\title{
Image Retrieval Using Mixture Models and EM Algorithm
}

\author{
Micheline Najjar $^{1}$, Christophe Ambroise $^{1}$, and Jean Pierre Cocquerez ${ }^{1}$ \\ Heudiasyc UMR CNRS 6599, Universite de technologie de compiegne, BP 20529, \\ 60205 Compiegne, France \\ \{Micheline.Najjar, Christophe.Ambroise, Cocquerez\}@hds.utc.fr
}

\begin{abstract}
This paper presents an original system for interactive content-based image retrieval (CBIR). A novel approach for searching by similarity is introduced. It is based on a classification of the index database using mixture models and the EM algorithm. The presented retrieval system is evaluated and validated using a medical image database and the Washington University heterogeneous database (ANN).
\end{abstract}

\section{Introduction}

The exponential development of computer power and the rise of information technologies have strongly supported the numerical image production. The processing of this data requires the development of effective procedures dedicated to information retrieval.

Several content-based image retrieval systems (CBIR) were developed during the last years (interested readers can refer to [9]) to deal with this problem. The majority are conceived for heterogeneous databases [4] (images concerning different topics). But the problem remains difficult, in particular in medicine where the medical image production is attached to various methods like the MRI (Magnetic Resonance Imaging), endoscopy, scintigraphy, etc., and the image analysis requires expert knowledge to detect and extract the physical signs characterizing pathological cases [8].

In this paper, we present a general image retrieval system based on a semi-supervised learning approach. This system was primarily dedicated to diagnosis help and applied to MRI medical image database.

The off-line indexing stage depends on the image database nature. The image index database is used online inside a relevant feedback loop which takes into account the physician knowledge.

To demonstrate that our image retrieval approach is generic, we applied it to another kind of index computed on images from the Washington University heterogeneous database (ANN). The index computation is described in [4], it is based on color and texture pixel features. Experimental results obtained with the two databases are presented in section 4 to assess the performance of the relevance feedback. Considering small databases is a deliberate choice, since we have a real medical application. Our main problem is the quality of the online diagnostic and not to deal with huge database.

J. Bigun and T. Gustavsson (Eds.): SCIA 2003, LNCS 2749, pp. 1114-1121, 2003.

(C) Springer-Verlag Berlin Heidelberg 2003 


\section{Presentation of the image retrieval problem}

One of the principal difficulties of image retrieval by contents consists in filling the existing semantic gap between the high level information contained in the image, which is complex and subjective, and the low level feature used to index the images. The formalization of this problem generally results in the construction of a similarity measure between the images to be compared [9]. In the context of the image retrieval, the relevance feedback represents the part of the system which, in an iterative way, integrates user knowledge in the definition of the similarity.

Among the possible definitions of the similarity measure (or in an equivalent way, dissimilarity measure), an approach consists in building a metric which is used to define the distance between the indexes. For instance, let us quote the approach based on the fuzzy logic suggested by Santini and al. [7] or that of Fournier and al. [4] which adapts the parameters of their L-metric by minimizing in an adaptive way a criterion, function of the distances between the image query and the images returned by the user.

Another way of dealing with the problem of searching for images by similarity consists in regarding it as a statistical database learning problem. Within this framework, two classes are considered: relevant images (chosen by the user) and irrelevant images. The learning problem thus consists in estimating the parameters of a classifier which distinguishes these two classes of images "as well as possible" and attributes a degree of relevance to each database image. Let us note that many approaches of image indexing based on similarity measure construction can be regarded as approximations or particular cases of well-known classification algorithms. The characteristics of the image retrieval problem compared to a standard classification are: the very low number of annotated images at the beginning of the search (small size of the training set), the high dimension of the index (a significant number of features) and the incremental definition of the training set. Moreover parameters of the classifier must be updated "quickly" after each new relevance feedback. Our work is within this framework.

\section{Semi-supervised retrieval approach based on mixture models}

In the following section we present our contribution to image retrieval. The proposed system comprises a relevance feedback based on a mixture model classifier whose parameters are estimated by an extended EM algorithm. This type of approach was applied by Y.Wu and al in [10] describing a modified EM algorithm which classifies the data, after a projection by linear discriminant analysis, in a subspace of dimension $(K-1)$, where $K$ is the number of considered classes. Y.Wu and al. suggest that this projection allows to avoid the assumption of the classes Gaussian distribution in the initial space. Let us note that in their approach, the indexes are often projected on a straight line, which over-simplifies the structure of the index database. Thus, this type of projection does not allow to model complex structures of classes such as a configuration where the class of the relevant images would be surrounded by the class of the irrelevant images. The approach we present appears more flexible because we suppose that the distribution of each class is itself a mixture of distribution (Gaussian for example, but not necessarily). This allows to capture complex structures and to better adapt to the richness of the user knowledge. 


\subsection{Basic formulation}

Let $\mathbf{D}$ be the set of indexed images annotated or not:

$$
\mathbf{D}=\left\{\left(\mathbf{x}_{1}, \mathbf{z}_{1}\right), \ldots,\left(\mathbf{x}_{N}, \mathbf{z}_{N}\right)\right\},
$$

where $\mathbf{x}_{i}$ is the index considered as a real vector of dimension $d$ and $\mathbf{z}_{i}$ is an annotation vector such as $\mathbf{z}_{i} \in\{0,1\}^{K}$ with $K$ is the number of image classes and:

- $z_{i k}=1$ means that $\mathbf{x}_{i}$ may belong to the class $k$,

- $z_{i k}=0$ means that $\mathbf{x}_{i}$ does not belong to the class $k$.

The vector $\mathbf{z}_{i}$ allows to represent the indetermination of membership of an image to a class when all its components are equal to one. When only one component of index $k$ is equal to 1 , the image is known to belong to a unique class $k$. When more than one component is set to one, and at least one other component equals to zero, the image may belong to several classes and we consider the image as annotated. We are thus able to code complex information. Note that this modelling assumes that the user holds the truth and is never mistaken in annotating an image.

$\mathbf{D}$ is considered as a sample of $N$ independent random vectors $(\mathbf{X}, \mathbf{Z})$. The problem consists in finding the label $y_{i} \in[1, \ldots, K]$ of the vector $\mathbf{x}_{i}$.

The indexes are supposed to be generated by a mixture of distributions:

$$
f\left(\mathbf{x}_{i} ; \phi\right)=\sum_{k=1}^{K} p_{k} \cdot f_{k}\left(\mathbf{x}_{i} ; \theta_{\mathbf{k}}\right)
$$

where $K$ is the number of mixture components (we suppose for the moment that each class is made of one component), $f_{k}$ is the probability density of parameter $\theta_{k}, p_{k}$ is the proportion of the component $k$ in the mixture model, and $\phi=\left(p_{1}, \ldots, p_{K} ; \theta_{1}, \ldots, \theta_{K}\right)$.

The label $y_{i}$ indicates from which component $\mathbf{x}_{i}$ is resulting. We can then write $\left(y_{i} / \mathbf{x}_{i}\right)$ as following a multinomial distribution:

$$
p\left(y_{i}=k / \mathbf{x}_{i}, \mathbf{z}_{i} ; \phi\right)=\frac{z_{i k} \cdot p_{k} \cdot f_{k}\left(\mathbf{x}_{i} ; \theta_{k}\right)}{\sum_{l=1}^{K} z_{i l} \cdot p_{l} \cdot f_{l}\left(\mathbf{x}_{i} ; \theta_{l}\right)} .
$$

The parameters of the mixture model can be estimated by the EM algorithm (Expectation Maximization, [3]). The alternative EM algorithm used in the relevance feedback was proposed by Ambroise and Govaert [1] to take into account annotated data using mixtures models. The semi-supervised learning is a particular case where the examples are classified or undetermined. [6] used the semi-supervised learning with the EM algorithm to classify texts and highlighted the interest to use undetermined (unlabelled) data to estimate the parameters of a classifier when classified data (labelled) is rare.

The conditional probability of equation 3 is used as a similarity measure between the query image index $\mathbf{x}_{r}$ and other database images. 


\subsection{Extension of the Model: multiple components by class}

An interesting extension of the previously described model consists of fitting multiple components to each class (MDA, [5]). In this paper the two class case is considered and the global probability density function of the observations is formed respectively of $K_{1}$ and $K_{2}$ components:

$$
f\left(\mathbf{x}_{i} ; \phi\right)=\sum_{k_{1}=1}^{K_{1}} p_{k_{1}} \cdot f_{k_{1}}\left(\mathbf{x}_{i} ; \theta_{k_{1}}\right)+\sum_{k_{2}=K_{1}+1}^{K_{1}+K_{2}} p_{k_{2}} \cdot f_{k_{2}}\left(\mathbf{x}_{i} ; \theta_{k_{2}}\right) .
$$

In this case the annotation vector is defined by $\mathbf{z}_{i} \in\{0,1\}^{K_{1}+K_{2}}$ and the label $y_{i}$ takes its value in $[1,2]$ :

$-y=1$ is coded by $\mathbf{z}=(\underbrace{1, \ldots, 1}_{K_{1} \text { times }}, \underbrace{0, \ldots, 0}_{K_{2} \text { times }})$

$-y=2$ is coded by $\mathbf{z}=(\underbrace{0, \ldots, 0}_{K_{1} \text { times }}, \underbrace{1, \ldots, 1}_{K_{2} \text { times }})$,

$-\mathbf{z}=(\underbrace{1, \ldots, 1}_{K_{1}+K_{2} \text { times }})$ means that no information about the relevance of the corresponding image is available.

\subsection{Relevance feedback and advantages of the method}

Our application requires only two classes, class $P$ of the relevant images and class $N$ of the irrelevant images. When the user chooses his query image, the EM algorithm described previously estimates the parameters of the distribution by using the query image as the mean vector of class $P$. All other images of the database are thus undetermined but used by the classifier to estimate the parameters of the mixture. Following the parameters estimation, the conditional probabilities of memberships of images to class $P$ (equation 3) are computed and $N_{S}$ images are displayed on the screen by decreasing order of the conditional probability. The user classifies among the $N_{S}$ images, those which are of interest to him (relevant images), the others being automatically classified as irrelevant. Each relevance feedback refines the query by re-estimating the parameters of the model with the new annotations. The suggested approach presents certain advantages compared to existing work:

- Considering the limited number of images which can be presented to the user or the small number of annotated images compared with those undetermined, the use of undetermined (unlabelled) images in the relevance feedback to refine the search reduces classification errors [6],

- allowing multimodal classes offers an acceptable solution to model the complexity of the borders between relevant and irrelevant image classes. It seems for example rather natural to regard the class of the relevant images as a complex category and to model it by a mixture of densities (see section 4.1). 


\section{Experimental results}

This section aims at giving some elements of validation of our general retrieval system to search for similar images. The relevance feedback quality is assessed using two different databases: 1)images of osteo-articular radiology (Section 4.2) and 2) the Washington University heterogeneous database (ANN, Section 4.3).

To quantify the retrieval effectiveness of our system, we used two well known and relevant quality criteria (see [9]) precision and recall. Since there is no optimal value for the cardinality of the retrieved images set, the two criteria are usually presented as a function of the number of retrieved images. The precision curve versus the recall ( $P R$ curve) is also meaningful. For example, low precision at high recall indicates that the system has difficulty in capturing the diversity of the relevant images. To assess the performance of the relevance feedback, the curve $P R$ is plotted for various numbers of feedback. For a given image category in the database, the quality criteria are calculated for several random requests and averaged over all these queries.

\subsection{Specification of the model}

Practically, as regards the implementation of the method presented in section 3, we suppose that the relevant images (class $P$ ) follow a mixture of two multidimensional Gaussian densities, with diagonal variance covariance matrix. This latter assumption allows to reduce the number of parameters to be estimated (but always keeps the possibility of a complex class) and to thus increases the robustness of the method. The irrelevant images can be seen as noisy observations which are disturbing the search for relevant images. For that we have modelled the irrelevant class $N$ like Uniform distribution over a volume including all observations. In this case, we only need to estimate one additional parameter (the proportion $p_{3}$ ) and the equation 2 becomes:

$$
f\left(\mathbf{x}_{i} ; \phi\right)=\sum_{k=1,2} p_{k} \cdot \frac{(2 . \pi)^{-\frac{d}{2}}}{\operatorname{det}\left|\Sigma_{k}\right|^{\frac{1}{2}}} \cdot e^{-\frac{1}{2}\left(\mathbf{x}_{i}-\mu_{\mathbf{k}}\right)^{t} \mathbf{\Sigma}_{\mathbf{k}}{ }^{-1}\left(\mathbf{x}_{i}-\mu_{\mathbf{k}}\right)}+p_{3} \cdot \frac{1}{V}
$$

$\mu_{\mathbf{k}}$ and $\Sigma_{\mathbf{k}}$ are the mean vector and the variance covariance matrix of the class $P . V$ is the domain volume of the indexes.

Choosing two component for modelling the relevant image class is not a limitation of our approach. Further work will propose a strategy to automatically find the best number of component.

\subsection{Medical Application}

In this section, we present searching results in the osteo-articular radiology image database [2]. After an automatic segmentation based on the level set approach which is introduced by Osher and Sethian [11], every image is represented by a set of labelled regions, each one characterized by a feature vector. Then, an unsupervised classification of the regions, using mixture models and the EM algorithm, is performed using a 
number of images randomly selected from the database. Thus the index of an image $i$ is noted by $\mathbf{x}_{\mathbf{i}}=\left\{x_{i j} ; 1 \leq j \leq d\right\}$. It is defined in the following way:

$$
x_{i j}=\frac{\sum \text { surfaces of the regions } \in C_{j}}{S_{\text {object }}},
$$

with $S_{\text {object }}$ is calculated by: $S_{\text {object }}=S_{\text {image }}-S_{\text {background }}$, and $C_{1}, C_{2}, \ldots, C_{d}$ are the region classes which are of number $d$.

The considered database is made up of 122 images of which 74 are normal hands and 39 relate to pathological or abnormal hands. The last nine parasitic images (feet, other images of the sequence of the MRI images for hands) were added to the base to test the robustness of the segmentation and the effectiveness of the search for similar images. The curves of the two quality criteria, according to the number of retrieved images $N_{S}$ show variations from 2 up to 122 . They are presented in figure 1 regarding the search for normal and abnormal images for various iteration counts of the relevance feedback. On the curve of recall in function of $N_{S}$ plotted for the category of the normal images (cf. figure 1), we notice that the recall reached its maximum of 1 with the fourth iteration for a number of retrieved images equal to or higher than 74 (the number of normal images in the database). That means that for the normal image query search, the system returns, at the fourth iteration, images which are all normal, whatever their number in the database. That explains also the behavior of the precision of the fourth iteration, which is close to 1 for a number of retrieved images lower than 74 , which means that four iterations are enough to reach the possible maximum precision (as many relevant retrieved images as existing relevant images). Results of the same type, although slightly less efficient ( $90 \%$ instead of the $100 \%$ previously noted), are observed for the two computed criteria on the abnormal images.

\subsection{ANN Image database}

We have tested our retrieval system on the Annotated ground-truth database (ANN) ${ }^{1}$ which provides 493 photographs of different topics. Indexes used in this experiments were computed by Jérôme Fournier ${ }^{2}$ thanks to the approach proposed in [4] for RETIN retrieval system. The image signature computed is an histogram of pixel features obtained via an unsupervised classification.

To improve the effectiveness of our relevance feedback, we plotted the curves of precision and recall (see figure 2). They are presented for the two categories "Arbogreens" and "Campusinfull" and for increasing numbers of feedback iterations. The evolution of the curve $P R$ (precision vs. recall) for Campusinfull category traced without feedback until this plotted after four feedback iterations shows that the retrieval performance increase. Moreover, for 30 retrieved images, precision increases $17.41 \%$ after one feedback step and $42,41 \%$ after four steps. However results of this evaluation procedure for the retrieval process are dependent on the ground-truth annotations and the richness of the image signature.

\footnotetext{
1 "Annotated groundtruth database", Departement of Computer Science and Engineering, University of Washington,http://www.cs.washington.edu/research/imagedatabase/.

${ }^{2}$ Equipe de Traitement des Images et du Signal, University of Cergy-Pontoise / ENSEA, 6 av. du Ponceau 95014 Cergy-Pontoise Cedex, France
} 


\section{Conclusion}

In this paper an original image retrieval system is presented. We consider the retrieval problem in a pattern recognition framework. Using mixture models and EM algorithm, a classifier is estimated for each user request, considering that there are two classes of images: relevant and irrelevant images. An extension to the EM algorithm for mixture models was proposed to obtain complex structures of classes. The proposed method is illustrated successfully on two image databases. It shows that the proposed system is able to deal with different type of images. As the number of images presented to the user is really small, the main advantage of the proposed method consists in using all database images in the relevance feedback to estimate the classifier parameters. The direct future work prolonging this research are as follows: A comparison between the presented system and existing systems using different evaluation procedures. In particular, it would be interesting to compare our method under certain assumptions and the approaches based on a geometrical design of the definition of similarity. In addition to the above, the online features selection will be very important to 1) respond the user knowledge and 2) the classifier estimation especially in case of high dimension indexes.

\section{References}

1. C. Ambroise, G. Govaert, "Em algorithm for partially known labels". IFCS2000, 7th Conference of the International Federation of Classication Societies, Namur, Belgique, pp.11-14, 2000.

2. Boutry, N., Lard, A., Solau, E., Flipo, R., Cotten, A.: The Usefulness of MR Imaging in Early Rheumatoid Arthritis. RSNA, Chicago (2001) 25-30

3. A. Dempster, N. Laird, D. Rubin, "Maximum likehood from incomplete data via the EM algorithm”, Journal of the Royal Statistical Society, Vol 39, pp .1-38, 1977.

4. J. Fournier, M. Cord, S.F. Philipp, "Retin: A content-based image indexing and retrieval system”, Pattern Analysis and Applications Journal, Special issue on image indexation, Vol. 4, n 2/3, pp.153-173, 2001.

5. T. Hastie, R. Tibshirani, "Discriminant Analysis by Gaussian Mixtures", Journal of the Royal Statistical Society B, Vol. 58, pp. 155-176, 1996.

6. K. Nigam, A. McCallum, S. Thrun, T. Mitchell, "Text Classification from Labeled and Unlabeled Documents using EM", Machine learning, Vol.39, n2/3, pp. 135-167, 2000.

7. S. Santini, R. Jain, "Similarity Measures", IEEE T-PAMI, Vol. 21, n 9, 1999.

8. C. Shyu, C. Brodley, A. Kak, A. Kosaka, "Assert: A Phisician-in-the-Loop Content-Based Retrieval System for HRCT Image Databases", Computer Vision and Image Understanding, Vol. 75, n 1/2, pp. 111-132, 1999.

9. A. Smeulders, M. Worring, S. Santani, A. Gupta, R. Jain, "Content-Based Image Retrieval at the end of the Early Years", IEEE transactions on pattern analysis and machine intelligence, Vol. 22, n12, 2000.

10. Y. Wu, Q. Tian, T. Huang, "Discriminant-EM Algorithm with Application to Image Retrieval”, Proc. Computer Vision and Pattern Recognition, pp. 222-227, 2000.

11. S. Osher, J. Sethian, : "Fronts Propagating with Curvature-Dependent Speed: Algorithms based on Hamilton-Jacobi Formulations". J. of Computational physics, Vol. 79, pp. 12-49, 1988. 

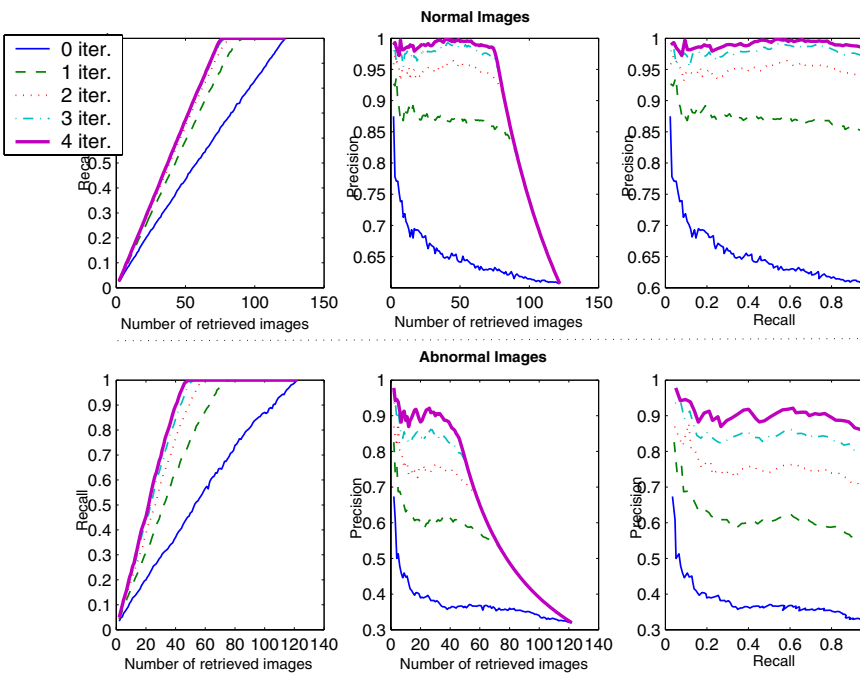

Fig. 1. System Evaluation on the medical images database
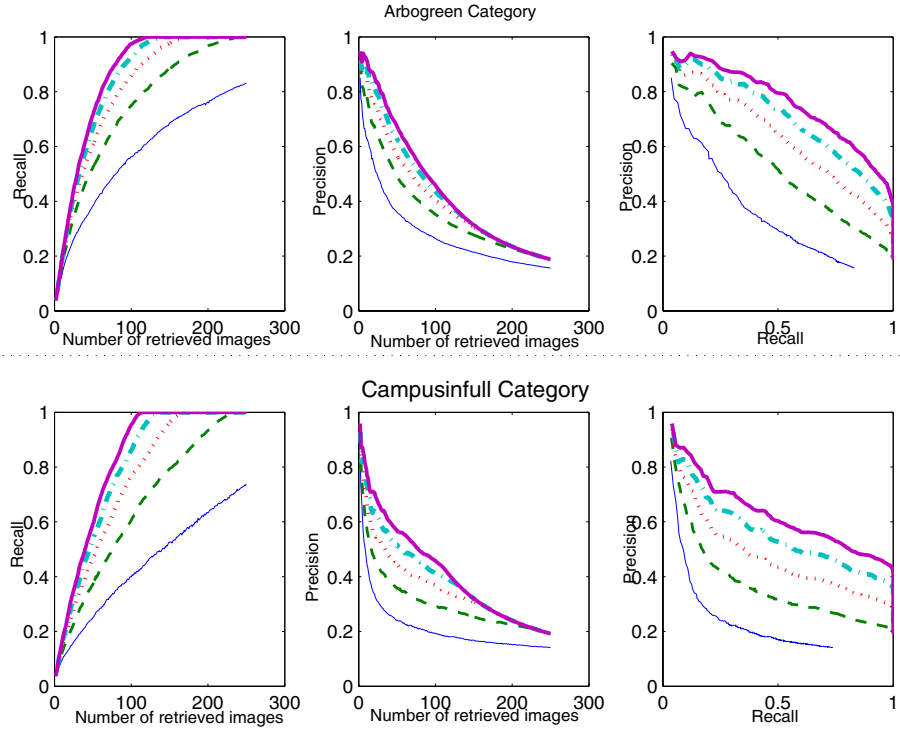

Fig. 2. System Evaluation on the ANN database 